\title{
Relationship between three-dimensional velocity of filament eruptions and CME association
}

\author{
Daikichi Seki ${ }^{1,2,34^{*}}$ D , Kenichi Otsuji ${ }^{5}$, Takako T. Ishii ${ }^{2}$, Ayumi Asai ${ }^{2}$ and Kiyoshi Ichimoto ${ }^{2}$
}

\begin{abstract}
It is widely recognised that filament disappearances or eruptions are frequently associated with Coronal Mass Ejections (CMEs). Since CMEs are a major source of disturbances of the space environment surrounding the Earth, it is important to investigate these associations in detail for the better prediction of CME occurrence. However, the proportion of filament disappearances associated with CMEs is under debate. The estimates range from $\sim 10$ to $90 \%$ and could be affected by the manners to select the events. In this study, we aim to reveal what parameters control the association between filament eruptions and CMEs. We analysed the relationships between CME associations and the physical parameters of filaments including their length, maximum ascending velocity, and direction of eruptions using 28 events of filament eruptions observed in $\mathrm{H} \alpha$. We found that the product of the maximum radial velocity and the filament length is well correlated with the CME occurrence. If the product is larger than $8.0 \times 10^{6} \mathrm{~km}^{2} \mathrm{~s}^{-1}$, the filament will become a CME with a probability of $93 \%$, and if the product is smaller than this value, it will not become a CME with a probability of $100 \%$. We suggest a kinetic-energy threshold above which filament eruptions are associated with CMEs. Our findings also suggest the importance of measuring the velocity vector of filament eruption in threedimensional space for the better prediction of CME occurrence.
\end{abstract}

Keywords: Filaments, Filament eruptions, Coronal Mass Ejections, Ha observation, 3D velocity

\section{Introduction}

Filaments are regions of dense cool plasma floating in the corona that are supported by magnetic fields. They are observed in absorption as dark features on the solar disk in $\mathrm{H} \alpha(6562.8 \AA)$ and in emission as prominences above the solar limb. At the end of its life, a filament disappears by slow fading or exhibits a transient eruption. Before it disappears or erupts, small-scale blobs observed in $\mathrm{H} \alpha$ in a filament often show a larger standard deviation of the line-of-sight (LOS) velocity (Seki et al. 2017, 2019b). During eruption phase, the entire body of a filament ascends at a velocity of $100-1000 \mathrm{~km} \mathrm{~s}^{-1}$ (Parenti 2014).

\footnotetext{
*Correspondence: seki.daikichi.87s@kyoto-u.jp

${ }^{1}$ Graduate School of Advanced Integrated Studies in Human Survivability, Kyoto University, Sakyo, Kyoto 606-8306, Japan

Full list of author information is available at the end of the article
}

Filament eruptions are often associated with coronal mass ejections (CMEs), which are observed by coronagraphs such as the Large Angle and Spectrometric Coronagraph (LASCO) (Brueckner et al. 1995). Some CMEs exhibit a three-part structure Illing and Hundhausen (1985) consisting of a leading edge, faint coronal cavity, and dense core. Others exhibit more complex forms, appearing as narrow jets or global eruptions, which are called halo CMEs (Webb 2015). The core of a CME is believed to originate from the filament mass if the CME is associated with a filament eruption. Because the Sun is concealed by an occulting disk in coronagraph observations of CMEs, it is difficult to observe the early evolution of CMEs. Investigations of the evolution of filament eruptions and their association with CMEs are expected to clarify the early evolution of CMEs.

CMEs often produce severe geomagnetic storms, which expose the Earth to a potential risk of adverse 
socioeconomic impacts such as a widespread blackout (Boteler 2001). A CME associated with a polar crown filament eruption reportedly caused a severe geomagnetic disturbance (Dst $\sim-200 \mathrm{nT}) 3$ days after the eruption (McAllister et al. 1994, 1996). Therefore, to mitigate the socioeconomic impacts of geomagnetic disturbances, it is essential to predict the occurrence of CMEs and their arrival to the Earth. And to reveal the relationship between CMEs and the eruption or disappearance of filaments is important for the better prediction.

However, the reported proportion of filament disappearances or eruptions that are associated with CMEs ranges from $\sim 10$ to $\sim 90 \%$. Hori and Culhane (2002) studied 50 prominence eruptions observed at $17 \mathrm{GHz}$ by the Nobeyama Radioheliograph (Nakajima et al. 1994) and found that $92 \%$ of them were associated with CMEs. Seki et al. (2019a) investigated 43 filament disappearances in $\mathrm{H} \alpha$ data observed by the Solar Dynamics Doppler Imager (SDDI) (Ichimoto et al. 2017) on the Solar Magnetic Activity Research Telescope (SMART) (UeNo et al. 2004) at Hida Observatory, Kyoto University, and found that $50 \%$ of them were associated with CMEs. McCauley et al. (2015) studied 904 filament and prominence eruptions observed in $\mathrm{He}(\mathrm{II})$ (304 $\AA$ ) by the Atmospheric Imaging Assembly (AIA) (Lemen et al. 2011) and found that $73 \%$ of them were associated with CMEs. In contrast, Al-Omari et al. (2010) automatically classified 7332 filament/prominence eruptions reported by the National Centers for Environmental Information ${ }^{1}$ as events associated or not associated with CMEs and found that only $17 \%$ of them were associated with CMEs. [For a more detailed summary of previous studies on the filament-CME association, see Table 1 in Al-Omari et al. (2010)]. It is supposed that the discrepancy among these results could depend on how to select the events.

In this study, we aim to reveal what parameters control the association between the filament eruptions and CMEs. We investigate the relationships between physical parameters that characterise filament eruptions, i.e., the length, velocity during eruption, and direction of eruption, and the CME association. Several studies have shown that these parameters are well correlated with the CME association of filament eruptions (Seki et al. 2019a; Gilbert et al. 2000; Morimoto and Kurokawa 2003). Gilbert et al. (2000) studied 54 prominence eruptions observed above the limb in $\mathrm{H} \alpha$. They defined "eruptive prominences" as those in which all or part of the material escaped from the solar gravitational field and "active prominences" as those in which none of the material

\footnotetext{
${ }^{1}$ ftp://ftp.ngdc.noaa.gov/STP/SOLAR_DATA/SOLAR_FILAMENTS/ accessed in 2008.
}

appeared to escape. They found that eruptive prominences clearly had a larger apparent velocity (the velocity projected on the plane of the sky) above 1.10 solar radii than active prominences did and that eruptive prominences were more strongly associated with CMEs (94\%) than active ones (46\%). Our previous study (Seki et al. 2019a) found that filament eruptions are more likely to be associated with CMEs if the filament length exceeds 150 $\mathrm{Mm}$, the maximum radial velocity exceeds $140 \mathrm{~km} \mathrm{~s}^{-1}$, or their direction is inclined by less than $48^{\circ}$ with respect to the solar normal. Thus, in the present study, we focus on these three parameters of filament eruptions and investigate how the association rate varies with respect to them. Note that, in contrast to our previous work that we investigated the tendency of CME association with respect to individual physical parameters, the present study aims to improve the predictability of CME association by combining those parameters. In "Data" section, we provide a description of the data we utilised. In the succeeding section, the results will be provided, followed by summary and discussion.

\section{Data}

We selected events from the SMART/SDDI Filament Disappearance Catalogue ${ }^{2}$ (hereafter, the catalogue) (Seki et al. 2019a). The unique advantage of the SDDI is its wide wavelength coverage, which makes it possible to determine the LOS velocity of erupting filaments up to $400 \mathrm{~km} \mathrm{~s}^{-1}$. The catalogue lists 43 filament/prominence disappearances observed by SDDI from 2016 May 1 to 2019 June 18 , in which filaments/prominences totally disappeared at the $\mathrm{H} \alpha$ line centre. We selected 28 of these events that had a credibility value of 2 or 3 for CME association in the catalogue (description of "credibility" will be provided later). That is, we used only events whose CME association or non-association is fairly clear. Note that some of the events were excluded from our analysis, even though their credibility was 2 or 3 , because (1) terrestrial clouds covered the target filaments, and it was impossible to estimate their precise LOS velocities (Nos. $001,007,018,021,022,035$, and 043), or (2) the length of the target filament could not be measured due to the lack of observation (No. 029). Most of the selected events (26 of 28) are filament eruptions, and two events (on May 24 and June 20 in 2016) are prominence eruptions observed on the solar limb. Hereafter, we refer to these 28 events simply as filament eruptions.

The credibility value indicates how credible the CME association of an event is. We made a movie containing solar full-disk images of each event in the $\mathrm{H} \alpha$ line centre

\footnotetext{
${ }^{2}$ https://www.kwasan.kyoto-u.ac.jp/observation/event/sddi-catalogue/.
} 
Table 1 Criteria for determining the credibility of a CME association on the basis of $\Delta T$ and $\Delta \phi$

\begin{tabular}{|c|c|c|c|c|}
\hline & $\Delta T<2$ & $2<\Delta T<6$ & $6<\Delta T<9$ & $9<\Delta T$ \\
\hline$\Delta \phi<30$ & $\begin{array}{l}\text { with CME } \\
\text { credibility } 3\end{array}$ & $\begin{array}{l}\text { with CME } \\
\text { credibility } 2\end{array}$ & $\begin{array}{l}\text { without CME } \\
\text { credibilitv } 2\end{array}$ & $\begin{array}{c}\text { without CME } \\
\text { credibility } 3\end{array}$ \\
\hline $30<\Delta \phi$ & $\begin{array}{l}\text { with CME } \\
\text { credibility } 2\end{array}$ & $\begin{array}{l}\text { with CME } \\
\text { credibility } 2\end{array}$ & $\begin{array}{c}\text { without CME } \\
\text { credibility } 2\end{array}$ & $\begin{array}{l}\text { without CME } \\
\text { credibility } 3\end{array}$ \\
\hline $70<\Delta \phi<1$ & $\begin{array}{l}\text { without CME } \\
\text { credibility } 2\end{array}$ & $\begin{array}{l}\text { without CME } \\
\text { credibility } 2\end{array}$ & $\begin{array}{c}\text { without CME } \\
\text { credibility } 2\end{array}$ & $\begin{array}{c}\text { without CME } \\
\text { credibility } 3\end{array}$ \\
\hline $100<\Delta \phi$ & $\begin{array}{c}\text { without CME } \\
\text { credibility } 3\end{array}$ & $\begin{array}{c}\text { without CME } \\
\text { credibility } 3\end{array}$ & $\begin{array}{c}\text { without CME } \\
\text { credibility } 3\end{array}$ & $\begin{array}{c}\text { without CME } \\
\text { credibility } 3\end{array}$ \\
\hline
\end{tabular}

The units of $\Delta T$ and $\Delta \phi$ are hour and deg, respectively

or in $304 \AA$ and LASCO C2 running difference images. While watching each movie, we examined the directional and temporal association of each filament eruption with the CME and assigned a credibility value based on our judgement. The actual movies used for examination can be accessed at the catalogue webpage (click the credibility column). Our judgement is based on (1) $\Delta T$, which is the difference between the time when a CME was first observed in LASCO and the time of total disappearance of the filament in $\mathrm{H} \alpha$ centre (same as FD_end_time in the catalogue), and (2) $\Delta \phi$, which is the difference between the position angle of the filament and the central position angle of the CME. Table 1 describes how we determined the credibility 2 or 3 of the CME association on the basis of $\Delta T$ and $\Delta \phi$. The credibility 1 was labeled to events which were difficult to determine one-to-one correspondence. More specifically, we labeled the credibility 1 (1) if two filaments disappeared within $1 \mathrm{~h}$, the difference between their $\Delta \phi^{\prime}$ 's was within $15^{\circ}$, and they were diagnosed as being associated with the same CME, or (2) if there were flares located within $30^{\circ}$ from the central position angle of the CME and within a few hours prior to CME occurrence. For example, the events No. 002 and No. 003 in the catalogue were categorised as the credibility of 1 because two filaments disappeared within $1 \mathrm{~h}$, and it was ambiguous which of these events was actually associated with one CME. Another example is No. 012, in which a CME could be attributable to a C-class flare occurred in an active region rather than to the filament eruption of the interest, and thus we concluded the credibility of this event as 1 .

Table 2 shows the selected 28 events with their CME associations and physical parameters.

'Date and time' is the start time of a filament eruption and is defined as the first observation of a dark feature in $\mathrm{H} \alpha-0.5 \AA$. It is equivalent to 'FD_start_time' in the catalogue. 'CME' indicates whether a filament eruption is associated with a CME. $V_{\mathrm{r}_{-} \max }, V_{\mathrm{r}_{-} \text {fin }}, V_{\text {pos }}$, and $V_{\max }$ are determined as follows. We manually tracked and measured the position and LOS velocity of a blob at the apex of the filament that was present until its total disappearance in $\mathrm{H} \alpha$. Then, we constructed its three-dimensional velocity as a function of time. $V_{\mathrm{r}_{-} \max }$ is the maximum radial (or ascending) velocity during the eruption, whilst $V_{\max }$ is the maximum magnitude of three-dimensional velocity. $V_{\text {pos }}$ is equal to $\sqrt{V_{x}^{2}+V_{x}^{2}}$, where $V_{x}$ and $V_{y}$ are the velocities of the filament in the east-west and south-north directions on the plane of the sky, at the time of $V_{\mathrm{r}_{-} \max }$, respectively. $V_{\mathrm{r}_{-} \text {fin }}$ is the radial velocity at the last observation of a filament in $\mathrm{H} \alpha . L$ is the length of a filament measured at the same time as 'date and time.' The projection effect is corrected according to the location of the filament on the solar disk. $\Theta$ is the inclination angle between the direction of the filament velocity at the time of $V_{\mathrm{r}_{-} \max }$ and the solar normal (see Figure B on the catalogue webpage). For further details of how these values were determined, see our previous paper (Seki et al. 2019a).

\section{Results}

Figure 1 displays the CME associations according to $L$ (vertical axis) and $V_{\mathrm{r}_{-} \max }$ (top left), $V_{\max }$ (top right), or $V_{\text {pos }}$ (bottom left) on a logarithmic scale.

Here, the length and velocities are normalised by $L_{0}=100 \mathrm{Mm}$ and $V_{0}=100 \mathrm{~km} \mathrm{~s}^{-1}$, respectively. We can see the tendency that the longer and faster filaments are more likely to be associated with CMEs. The solid lines in the panels are drawn by the following relationships;

$$
\begin{aligned}
& \left(\frac{V_{\mathrm{r}_{-} \max }}{V_{0}}\right) \times\left(\frac{L}{L_{0}}\right)^{0.96}=0.80, \\
& \left(\frac{V_{\max }}{V_{0}}\right) \times\left(\frac{L}{L_{0}}\right)^{1.1}=1.4,
\end{aligned}
$$


Table 2 Filament eruptions used in this study. Data are taken from the SMART/SDDI Filament Disappearance Catalogue (Seki et al. 2019a)

\begin{tabular}{|c|c|c|c|c|c|c|c|c|}
\hline $\begin{array}{l}\text { Date and time } \\
\text { (UT) }\end{array}$ & CME & Cred. $^{a}$ & $V_{r_{-} \max }\left(\mathrm{km} \mathrm{s}^{-1}\right)$ & $V_{r_{-} \text {fin }}\left(\mathrm{km} \mathrm{s}^{-1}\right)$ & $V_{\text {pos }}^{b}\left(\mathrm{~km} \mathrm{~s}^{-1}\right)$ & $V_{\max }\left(\mathrm{km} \mathrm{s}^{-1}\right)$ & $L(\mathrm{Mm})$ & $\Theta^{c}\left({ }^{\circ}\right)$ \\
\hline 2016-05-24 01:00 & Yes & 3 & 134 & 36.4 & 134 & 175 & 137 & 11.8 \\
\hline 2016-06-01 21:00 & Yes & 3 & 173 & 50.8 & 189 & 226 & 151 & 25.7 \\
\hline 2016-06-20 05:30 & Yes & 3 & 215 & 41.3 & 223 & 224 & 63.6 & 16.6 \\
\hline 2016-07-07 07:19 & Yes & 3 & 359 & 189 & 272 & 365 & 35.6 & 9.67 \\
\hline 2016-07-19 05:30 & No & 3 & 30.8 & -3.80 & 27.0 & 93.1 & 31.7 & 53.9 \\
\hline 2016-07-20 01:00 & Yes & 3 & 122 & -6.40 & 101 & 199 & 99.8 & 43.9 \\
\hline 2016-08-09 23:30 & Yes & 3 & 149 & 51.3 & 130 & 155 & 449 & 15.0 \\
\hline 2016-08-11 22:00 & No & 2 & 9.40 & 2.00 & 38.2 & 52.0 & 153 & 75.9 \\
\hline 2016-08-13 02:30 & No & 3 & 34.8 & -18.0 & 108 & 234 & 139 & 71.3 \\
\hline 2016-08-19 02:00 & No & 2 & 3.60 & 2.20 & 18.1 & 22.3 & 341 & 78.5 \\
\hline 2016-09-03 23:53 & No & 3 & 15.7 & 15.0 & 9.35 & 52.7 & 52.5 & 7.89 \\
\hline 2016-09-09 21:51 & No & 3 & 51.6 & 25.8 & 22.3 & 154 & 41.7 & 35.2 \\
\hline 2016-11-04 01:32 & Yes & 2 & 38.4 & 26.8 & 39.6 & 44.3 & 312 & 29.1 \\
\hline 2016-11-05 01:24 & Yes & 3 & 121 & 109 & 67.0 & 123 & 112 & 7.26 \\
\hline 2017-02-10 04:00 & No & 2 & 4.90 & -1.40 & 11.5 & 14.2 & 154 & 64.9 \\
\hline 2017-02-19 04:44 & Yes & 2 & 161 & 143 & 185 & 218 & 112 & 42.2 \\
\hline 2017-03-05 01:30 & No & 3 & 68.1 & 40.9 & 74.4 & 81.0 & 42.7 & 32.7 \\
\hline 2017-04-23 04:30 & Yes & 3 & 456 & 436 & 497 & 523 & 113 & 29.4 \\
\hline 2017-04-23 03:05 & No & 3 & 18.9 & -25.0 & 18.1 & 324 & 73.9 & 45.5 \\
\hline 2017-04-23 23:08 & Yes & 3 & 80.3 & 36.5 & 110 & 112 & 293 & 44.4 \\
\hline 2017-04-29 23:30 & Yes & 2 & 44.2 & -10.6 & 151 & 154 & 271 & 73.0 \\
\hline 2017-09-25 21:24 & Yes & 2 & 172 & 7.70 & 530 & 555 & 164 & 72.0 \\
\hline 2017-12-07 02:15 & No & 3 & 2.30 & -43.3 & 63.8 & 246 & 69.8 & 88.0 \\
\hline 2018-04-03 02:30 & No & 3 & 82.7 & -2.77 & 139 & 183 & 160 & 59.0 \\
\hline 2018-04-20 05:00 & No & 2 & 0.100 & -23.4 & 0.141 & 46.7 & 59.3 & 72.5 \\
\hline 2018-07-16 06:00 & No & 3 & 40.0 & -5.00 & 52.8 & 75.6 & 77.4 & 43.9 \\
\hline 2018-07-31 03:00 & No & 3 & 3.70 & 3.30 & 0.412 & 13.5 & 99.6 & 26.1 \\
\hline 2019-02-23 22:30 & Yes & 3 & 47.6 & 28.2 & 45.1 & 66.6 & 285 & 43.6 \\
\hline
\end{tabular}

a The credibility of the association between a CME and a filament eruption. $3>2>1$. Events with a credibility of 1 are excluded from this study.

${ }^{b}$ The apparent velocity of a filament. It is defined as $\sqrt{V_{x}^{2}+V_{y}^{2}}$, where $V_{x}$ and $V_{y}$ are the east-west and south-north velocities projected on the plane of the sky, respectively.

c The inclination angle of a filament eruption with respect to the solar normal

$$
\left(\frac{V_{\text {pos }}}{V_{0}}\right) \times\left(\frac{L}{L_{0}}\right)^{0.72}=0.85
$$

They were determined by using the algorithm of Linear Support Vector Classification implemented in LIBLINEAR (Fan et al. 2008) (for further explanation of the algorithm, see Appendix and Fan et al. 2008). In the top left panel $\left(V_{r_{-} \max }\right), 27$ events out of $28(96 \%)$ were correctly classified into the two groups of filament eruptions with (open circles) and without (crosses) CMEs, whilst in the other cases, 21 (for $V_{\max }$ ) and 25 ( $V_{\text {pos }}$ ) events were correctly separated. If we make a separation so that the number of correctly classified events can be maximised, $27\left(V_{\mathrm{r}_{-} \max }\right), 24\left(V_{\max }\right)$, and $26\left(V_{\text {pos }}\right)$ events will be correctly classified (the separations not shown in the figure). Thus, a better prediction of the CME association could be obtained by using $V_{\mathrm{r}_{-} \max }$ rather than using $V_{\max }$ or $V_{\text {pos }}$ at least with our limited number of the events, 28. This result suggests the advantage of measuring the radial velocity of filament eruptions. It also suggests that measuring both the velocity and the length of filaments should contribute to the better prediction of CME occurrence. The three-dimensional velocity observation provides a better capability for predicting the occurrence of CMEs, whilst the $\mathrm{H} \alpha$ imaging observations without Doppler measurements $\left(V_{\text {pos }}\right)$ can still contribute to it.

Figure 2 displays histograms of events with (grey) and without (black) CMEs with respect to the left-hand sides 

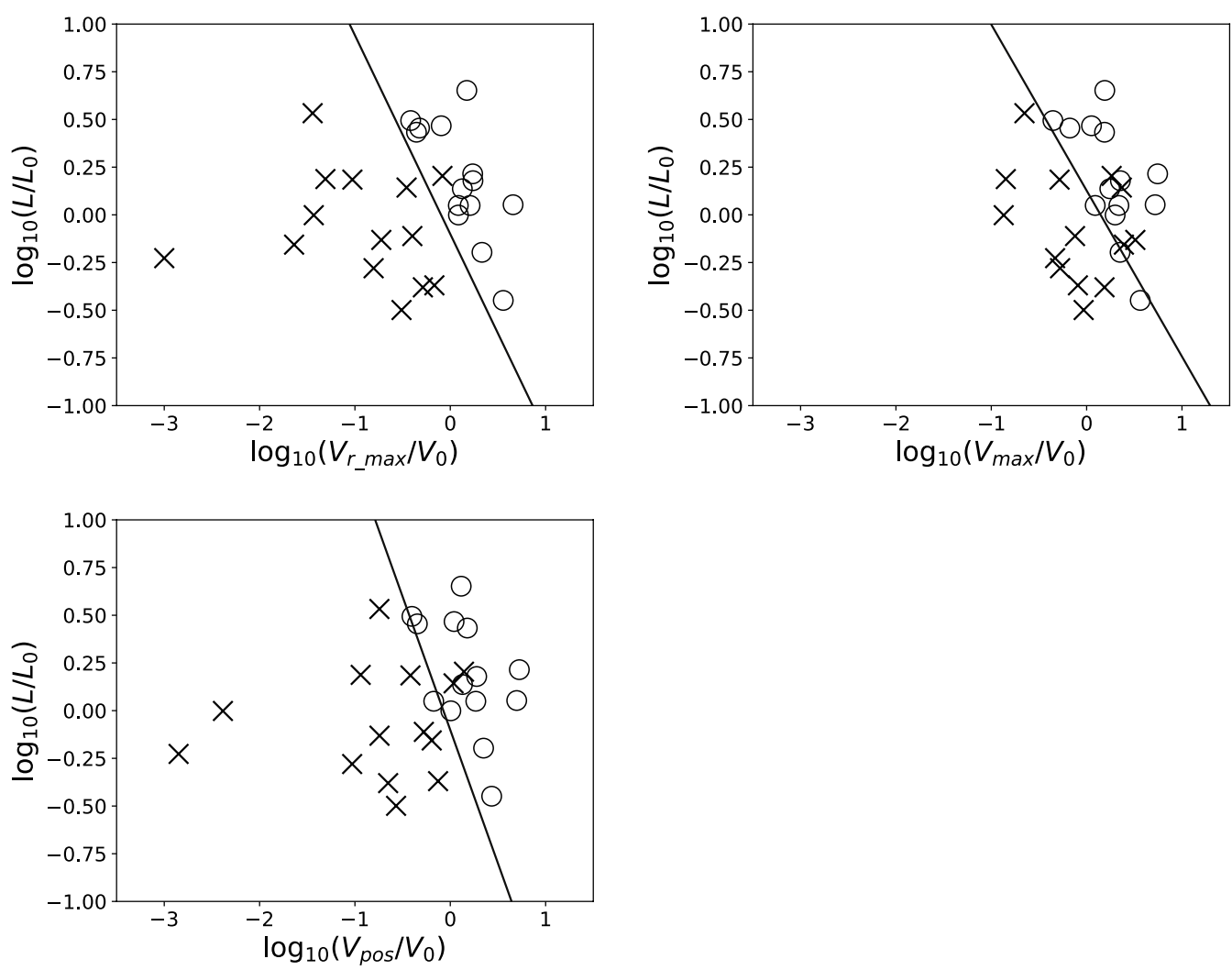

Fig. 1 Plots of filament eruptions according to $V_{r_{-} \max }$ (top left), $V_{\max }$ (top right), or $V_{\text {pos }}$ (bottom left) and filament length, $L$, on a common logarithmic scale. $V_{0}$ and $L_{0}$ correspond to the typical velocity $\left(100 \mathrm{~km} \mathrm{~s}^{-1}\right)$ and typical length $(100 \mathrm{Mm})$ of filaments, respectively. Open circles and crosses represent events with and without CMEs, respectively. The solid lines are described in the text

(LHS) of Eqs. (1)-(3). These histograms also demonstrate that the CME association is better identified when $V_{\mathrm{r}_{-} \max }$ is used than when $V_{\max }$ or $V_{\text {pos }}$ is used. In the top left panel, we see a clear bimodal distribution, which is less clear in the other cases. To confirm the bimodality quantitatively, we introduced a statistic, $D$-value, which is defined as

$$
D \equiv \frac{\left|\mu_{1}-\mu_{2}\right|}{\sigma}
$$

where $\mu_{1}$ and $\mu_{2}$ are the averages of the two normal distributions fitted to the events with and without CMEs, and $\sigma$ is equivalent to

$$
\sigma=\sqrt{\frac{\sigma_{1}^{2}+\sigma_{2}^{2}}{2}},
$$

where $\sigma_{1}$ and $\sigma_{2}$ are their standard deviations. The $D$-value represents the distance between the means of two normal distributions relative to their standard deviations. These distributions can be regarded as being separated if the $D$-value is larger than 2 (Ashman et al. 1994; Muratov and Gnedin 2010; Carlson et al. 2018). The means and standard deviations of two normal distributions (with and without CMEs) for each case of Eqs. (1)(3) are summarised in Table 3 together with the $D$-values. We obtained $D$ values of 2.3, 1.8, and 1.7 for $V_{\mathrm{r}_{-} \max }, V_{\max }$, and $V_{\text {pos }}$, respectively. Only $V_{\mathrm{r}_{-} \max }$ exhibits the $D$-value larger than 2 . The better bimodality when $V_{\mathrm{r}_{-} \max }$ is used is confirmed quantitatively.

Figure 3 shows the CME association with respect to the radial velocity of the last observation $\left(V_{\mathrm{r}_{-} \text {fin }}\right)$ and $L$ (left panel) or a common log of the LHS of Eq. (1) (right panel) for each filament eruption. Open circles and crosses denote events with and without CMEs, respectively. Most of the filament eruptions (80\%) with negative $V_{r_{-} \text {fin }}$ (grey area), i.e., events in which the filaments fall back to the Sun, were not associated with CMEs. In addition, $77 \%$ of the filament eruptions with positive $V_{\mathrm{r}_{-} \text {fin }}$ and $L$ larger than $70 \mathrm{Mm}$ were associated with CMEs. Note that the filaments with the smaller (larger) value of the LHS of Eq. (1) similarly tend to have smaller (larger) $V_{r_{-} \text {fin }}$ (see the right panel).

From Fig. 3, we can also recognise that there are exceptional events that were associated with CMEs despite their negative $V_{\mathrm{r}_{-} \text {fin }}$ 's $\left(-6.40 \mathrm{~km} \mathrm{~s}^{-1}\right.$ and -10.6 

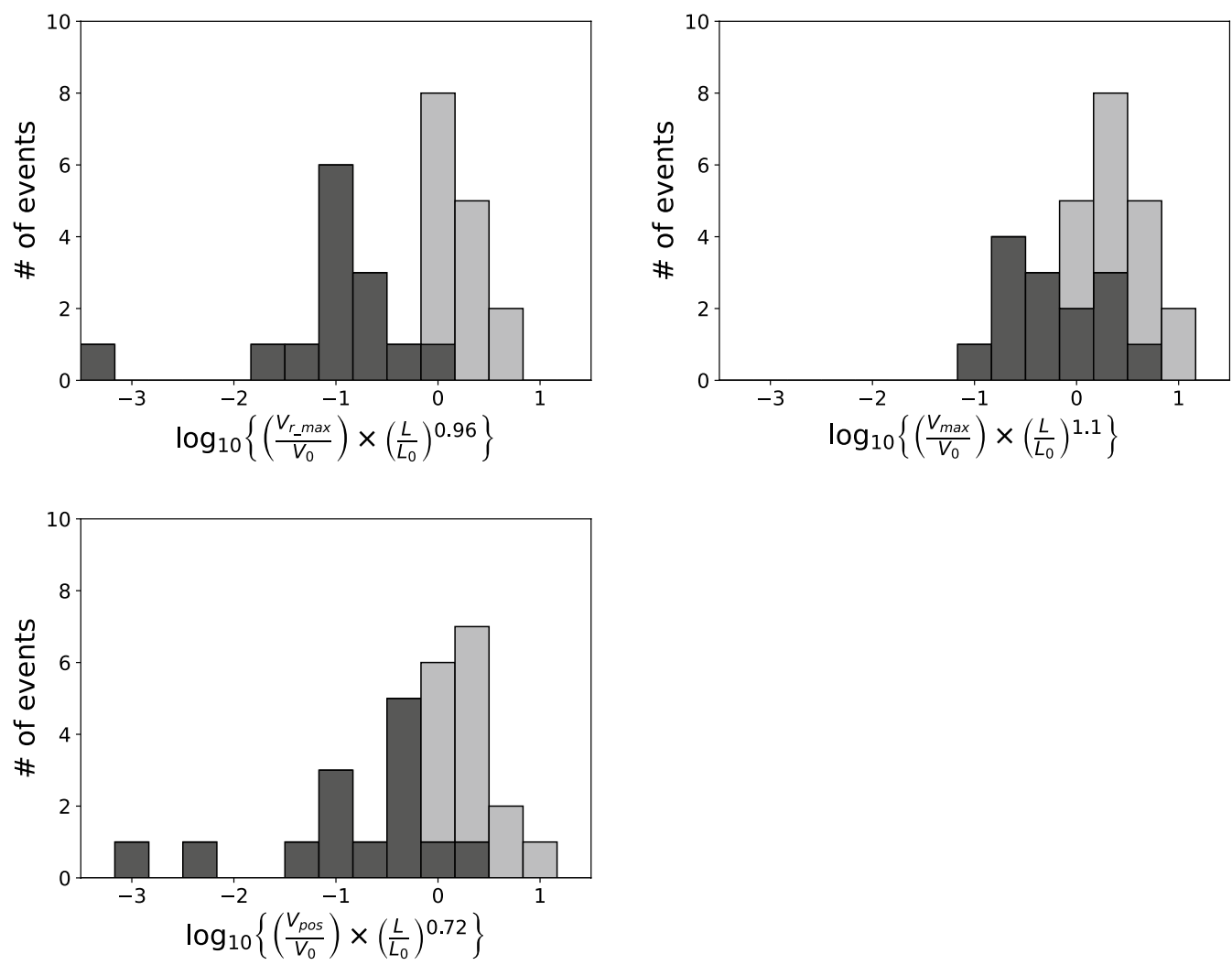

Fig. 2 Histograms of the LHS of Eqs. (1)-(3) on a common logarithmic scale. The dark and light grey bars correspond to the events without and with CMEs, respectively. The bars are stacked. The size of a bin is 0.33

Table 3 Summary statistics of the fitted normal distributions and the corresponding $D$ values

\begin{tabular}{|c|c|c|c|c|c|}
\hline & \multicolumn{2}{|c|}{ With CMEs } & \multicolumn{2}{|c|}{ Without CMEs } & \multirow[t]{2}{*}{$D$} \\
\hline & $\mu_{1}$ & $\sigma_{1}$ & $\mu_{2}$ & $\sigma_{2}$ & \\
\hline$V_{r_{-} \max }($ Eq. (1)) & 0.28 & 0.23 & -1.0 & 0.76 & 2.3 \\
\hline$V_{\max }($ Eq. (2)) & 0.46 & 0.28 & -0.20 & 0.44 & 1.8 \\
\hline$V_{\text {pos }}($ Eq. (3)) & 0.29 & 0.29 & -0.81 & 0.88 & 1.7 \\
\hline
\end{tabular}

$\mathrm{km} \mathrm{s}^{-1}$ ). We speculate that the blobs which escaped the solar gravity and erupted into the interplanetary space became invisible in $\mathrm{H} \alpha$, and we tracked a part of the filament that fell back to the solar surface.

Figure 4 shows the CME association according to the LHS of Eq. (1) (vertical axis) and $\Theta$, the inclination angle (angle from the solar normal) of the velocities (horizontal axis).We found that $82 \%$ of the filament eruptions with directions that were inclined by more than $45^{\circ}$ from the solar normal were not associated with CMEs, and $71 \%$ of those with their $\Theta$ 's smaller than $45^{\circ}$ were associated with CMEs. Thus, the inclination angle of eruptions will provide a clue for forecasting the CME occurrence. Note that the LHS of Eq. (1) of the filament eruptions with their $\Theta$ 's larger than $45^{\circ}$ seldom exceeds $-0.097(=\log 10(0.80))$. Figure 4 also shows that $86 \%$ of the events associated with CMEs have their $\Theta$ 's smaller than $45^{\circ}$, while only $36 \%$ of the non-associated ones have $\Theta$ smaller than $45^{\circ}$. These results are consistent with the work of Gopalswamy et al. (2003), in which they defined two types of prominence eruptions, radial and transverse events, according to the eruptive motion of the prominence observed on the solar limb. In their statistical study, they found that $94 \%$ of the prominence eruptions associated with CMEs were radial events ( $86 \%$ in our study) and that $76 \%$ of the transverse events were not associated with CMEs ( $82 \%$ in our study).

Figure 5 shows the linear speed of the CMEs in the SOHO/LASCO CME Catalog (Yashiro et al. 2004) against $V_{r_{-} \max }$.The CME speed is expected to be larger than the velocity of the associated filament eruption (Gopalswamy et al. 2003). The black line indicates equal speeds. All the data points but one $\left(V_{\mathrm{r}_{-} \max }=359 \mathrm{~km}\right.$ $\mathrm{s}^{-1}$ ) are above or close to the black line, as expected. However, since there is a large scatter in the ratio of CME linear speed to $V_{\mathrm{r}_{-} \max }$, it is difficult to predict CME linear speeds from the maximum radial velocity. 

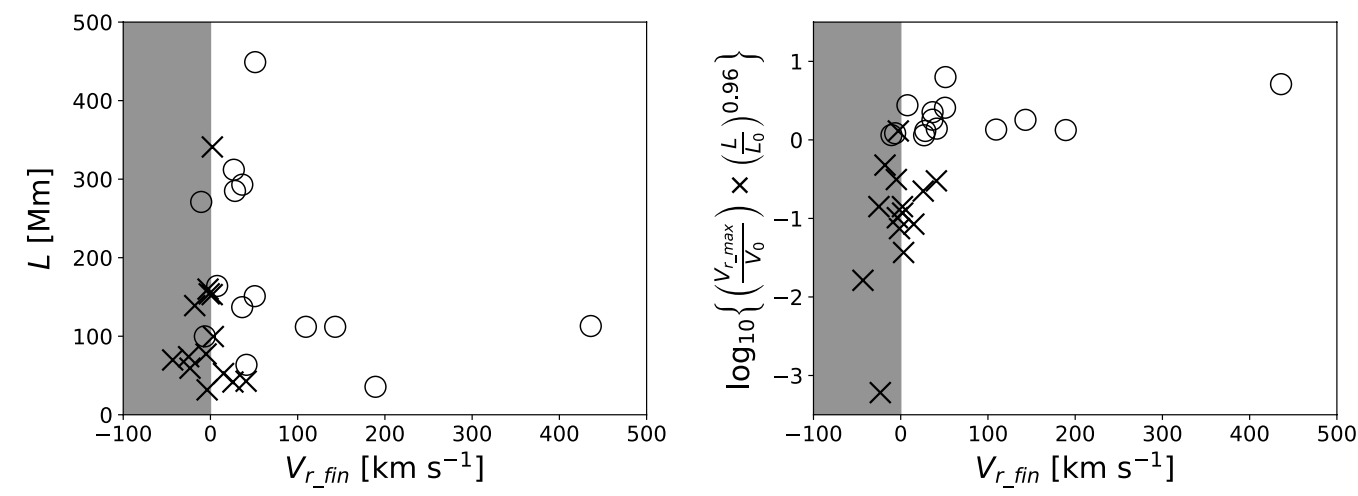

Fig. 3 Plot of filament eruptions according to $V_{r_{-} \text {fin }}$ and $L$ (left) or common $\log$ of the product of normalised $V_{r_{-} \text {max }}$ and normalised $L$ to the power of 0.92 (right). Open circles and crosses have the same meaning as in Fig. 1. Grey area corresponds to negative $V_{r_{-} \text {fin }}$

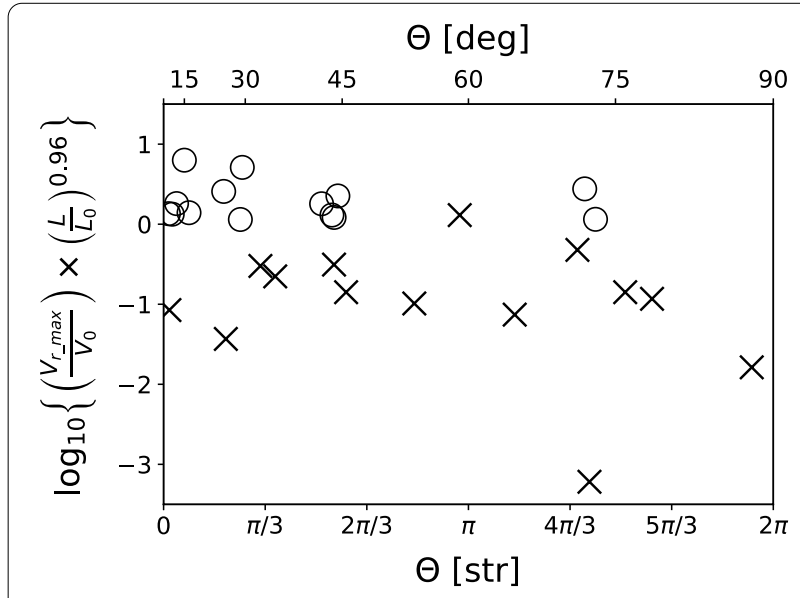

Fig. 4 Plot of filament eruptions according to $\Theta$ (inclination angle in steradians and degrees) and a common log of the LHS of Eq. (1). The symbols have the same meaning as in Fig. 1

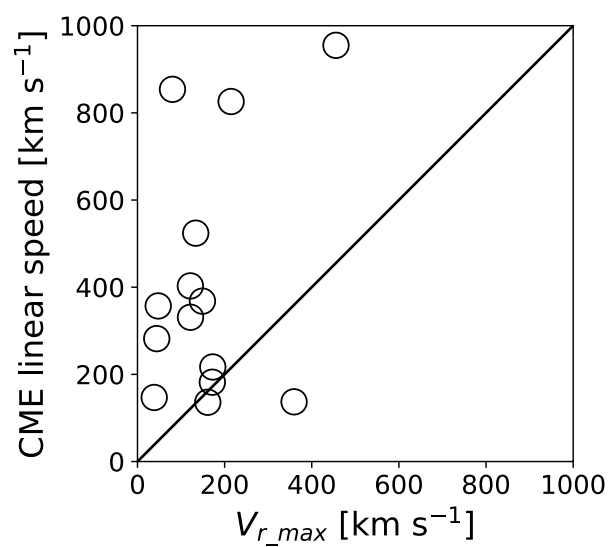

Fig. 5 Plot of filament eruptions according to $V_{r_{-} \max }$ and $C M E$ linear speed. The black line corresponds to equal speeds

\section{Summary and discussion}

In this study, we investigated the relationships between the physical parameters of filament eruptions (threedimensional velocity, filament length, and direction of eruption) and their CME associations using 28 events observed by SDDI at Hida Observatory. We found that the filament eruptions are well separated into two groups of events, one with and the other without CMEs, according to the product of the normalised maximum ascending velocity $\left(V_{\mathrm{r}_{-} \max } / V_{0}\right)$ and the normalised filament length $\left(L / L_{0}\right)$ to the power of 0.96 , and that among the filament eruptions with $\left(\frac{V_{\mathrm{r} \text { max }}}{V_{0}}\right) \times\left(\frac{L}{L_{0}}\right)^{0.96}>0.80,93 \%$ are associated with CMEs, and $100 \%$ of filament eruptions with the product $<0.80$ are not associated with CMEs. The apparent velocity and the length of filaments measured in $\mathrm{H} \alpha$ observation could also provide a good measure for predicting the occurrence of CMEs, though the accuracy of the prediction using the apparent velocity is worse than that using the radial velocity. Our results suggest that the three-dimensional velocity, or more specifically the radial velocity derived from it, and the length of the erupting filament are the notable parameters for improving the predictability of CME association. And thus, we suggests the importance of observations of the three-dimensional velocity of filament eruptions for the prediction of CMEs. It should be noted, however, that improvement of statistics, i.e., studies with a larger number of examples, are strongly required to confirm these results.

Here, we propose a possible physical interpretation for the solid line in the top left panel of Fig. 1. This line, which is represented by Eq. (1), successfully separates events into those with and without CMEs. We assume that (1) the cross section of filaments, $A$, follows the relationship of 


$$
\left(\frac{A}{A_{0}}\right)=\left(\frac{L}{L_{0}}\right)
$$

where $A_{0}$ is the typical cross section of filaments (100 $\mathrm{Mm}^{2}$ ), and that (2) the average hydrogen density is common among filaments, i.e., $10^{11} \mathrm{~cm}^{-3}$, which is a typical value for quiescent prominences (Heinzel et al. 2008). Then, if we regard Eq. (1) as

$$
\left(\frac{V_{\mathrm{r}_{-} \max }}{V_{0}}\right) \times\left(\frac{L}{L_{0}}\right) \sim 0.80,
$$

or $V_{\mathrm{r}_{-} \max } \times L \sim 8.0 \times 10^{6} \mathrm{~km}^{2} \mathrm{~s}^{-1}$, then its square represents the kinetic energy of an erupting filament, i.e., $\frac{1}{2} \times$ proton mass $\times$ density $\times$ volume $\times V_{\mathrm{r}_{-} \max }{ }^{2}=5.4 \times 10^{28}$ erg. This relationship could be regarded as the kineticenergy threshold above which filament eruptions are associated with CMEs. Note that if the length of a filament is $100 \mathrm{Mm}$, the deduced mass gets $1.7 \times 10^{15} \mathrm{~g}$. (Gilbert et al. 2006) reported the masses of 18 prominences, which ranged from $(1.08 \pm 0.52) \times 10^{14}$ to $(2.09$ $\pm 0.80) \times 10^{15} \mathrm{~g}$. Our assumed "typical" mass is consistent with the reported values.

As mentioned in "Introduction" section, the CME association rates of filament eruptions reported to date range from $\sim 10$ to $\sim 90 \%$. Here, we provide a possible interpretation of this wide range based on our results. We showed that the product of the normalised radial velocity of eruptions and the normalised filament length makes a key contribution to the CME association. The high association rates of $80-90 \%$ in the past studies might be attributable to the criteria they used to select the events, under which the prominences have a predominantly large radial velocity and a large size. Gilbert et al. (2000) reported that $94 \%$ of eruptive prominences (for the definition, see "Introduction" section) were associated with CMEs. Gopalswamy et al. (2003) also reported that $83 \%$ of radial prominence eruptions were associated with CMEs. Their selected prominence eruptions should have had a predominant radial velocity. In addition, Gopalswamy et al. (2003) and Hori and Culhane (2002) detected prominences with the NoRH that has its spatial resolution of $10 \operatorname{arcsec}$ (Nakajima et al. 1994), which is worse than the spatial sampling of the SDDI (1.23 $\left.\operatorname{arcsec}_{\text {pixel }}^{-1}\right)$. Therefore, the selected prominences in these studies seem to have a larger size (e.g., larger than $70 \mathrm{Mm}$, because $75 \%$ of the filaments smaller than $70 \mathrm{Mm}$ were not associated with CMEs according to our result).

The association rate could also depend on whether studies include disk events (filament disappearances) in the records. In contrast to the high association rates $(80$ to $90 \%$ ) reported in the studies taking into account only limb events (prominence disappearances) (Gilbert et al. 2000; Gopalswamy et al. 2003; Hori and Culhane 2002), some studies (Pojoga and Huang 2003; Jing et al. 2004; Seki et al. 2019a) in which both disk and limb events were considered manifested the association rate of approximately $40-50 \%$. Pojoga and Huang (2003) reported that $39 \%$ of filament and prominence eruptions observed in $\mathrm{H} \alpha$ were associated with CMEs. Jing et al. (2004) reported that $56 \%$ of filament eruptions were associated with CMEs by automatically detecting filament disappearances in $\mathrm{H} \alpha$. In our study, considering only credible events, we found that $50 \%$ of filament eruptions in $\mathrm{H} \alpha$ were associated with CMEs.

Additionally, the observational wavelengths at which filaments or prominences are detected could also affect the association rate. In $\mathrm{H} \alpha$, as mentioned in the previous paragraph, approximately 40 to $50 \%$ of disappearance events were associated with CMEs. By contrast, McCauley et al. (2015) used full-disk solar images in the 171, 193, and $304 \AA$ AIA passbands and reported an association rate of $72 \%$.

The low association rate (17\%, Al-Omari et al. 2010) might be attributable to the fact that the authors include ejecta such as surges in addition to filament eruptions in their sample. Among their 7332 events, they introduced 15 "filament types", including coronal rain, sprays, and surges. In our study, we did not refer to these ejecta as filaments, and we excluded them from our list. Thus, the definition of filaments in that study was different from ours. Moreover, most of their events $(\sim 80 \%)$ were smaller than $\sim 70 \mathrm{Mm}$ [see Figure 8 in Al-Omari et al. (2010)]. According to our result (see Fig. 1 or 3), $75 \%$ of the eruptions of filaments with lengths smaller than 70 $\mathrm{Mm}$ were not associated with CMEs. Assuming that this relation holds for coronal rain, surges, and sprays, $\sim 60 \%$ $(80 \% \times 75 \%)$ of all their selected events may not be associated with CMEs in our criteria. Therefore, the low association rate in their study can also be attributed to the event selection criteria; i.e., a significant portion of their events is thought to be located below the threshold line proposed in this paper.

The results of this study can be used to develop a methodology to predict the occurrence of CMEs by measuring the three-dimensional velocities of filament eruptions. Moreover, our previous works suggest that the occurrence of filament eruptions can be predicted prior to their initiation by $1.3 \pm 0.47$ hour for intermediate filaments, on the basis of the mean and standard deviation of the LOS velocity distribution in filaments (Seki et al. 2017, 2019b). Hence, by using SDDI data and measuring the LOS velocity of filaments, we could predict the occurrence of filament eruptions $\sim 1 \mathrm{~h}$ in advance and also, during eruptions, estimate the possibility of CME association before coronagraph observations. 


\section{Abbreviations}

CME: Coronal mass ejection; LASCO: Large Angle and Spectrometric Coronagraph; SMART: Solar Magnetic Activity Research Telescope; SDDI: Solar Dynamics Doppler Imager; AIA: Atmospheric Imaging Assembly; LOS: Line-of-sight; LSVC: Linear Support Vector Classification.

\section{Acknowledgements}

D.S. thanks anonymous reviewers for their precious comments. D.S. also thanks GSAIS Empirical Research Group (GERG) for the discussion to enrich the methodology. The SOHO/LASCO CME Catalog is generated and maintained at the CDAW Data Center by NASA and The Catholic University of America in cooperation with the Naval Research Laboratory.

\section{Authors' contributions}

DS: statistical analysis, structure and strategy of the paper, and writing the paper. OK: developing an algorithm to deduce three-dimensional velocity of filaments. TTI: observation and calibration. AA: structure and strategy of the paper. Kl: structure and strategy of the paper. All authors read and approved the final manuscript.

\section{Funding}

This work was supported by JSPS KAKENHI Grant Numbers JP15H05814 (Project for Solar-Terrestrial Environment Prediction, PSTEP), JP16H03955, and JP18J23112. D. S. is supported by Research Fellowships for Young Scientists from the Japan Society for the Promotion of Science.

\section{Availability of data and materials}

See https://www.kwasan.kyoto-u.ac.jp/observation/event/sddi-catalogue/.

Ethics approval and consent to participate

Not applicable.

\section{Consent for publication}

Not applicable.

\section{Competing interests}

The authors declare that they have no competing interests.

\section{Author details}

${ }^{1}$ Graduate School of Advanced Integrated Studies in Human Survivability, Kyoto University, Sakyo, Kyoto 606-8306, Japan. ${ }^{2}$ Astronomical Observatory, Kyoto University, Sakyo, Kyoto 606-8502, Japan. ${ }^{3}$ Department of Applied Mathematics and Theoretical Physics, University of Cambridge, Wilberforce Road, Cambridge CB3 OWA, UK. ${ }^{4}$ Centre for the Study of Existential Risk, University of Cambridge, 16 Mill Lane, Cambridge CB2 1SB, UK. ${ }^{5}$ Space Environment Laboratory, Applied Electromagnetic Research Institute, National Institute of Information and Communications Technology, Koganei, Tokyo 184-8795, Japan.

\section{Appendix: Linear Support Vector Classification (LSVC)}

Linear Support Vector Classification (LSVC), which we utilised to estimate the CME association from the observation of filaments, is one of the popular machine-learning methods for classification. Our goal is to obtain the coefficients of the solid lines in Fig. 1, which successfully separate the events associated with CMEs from those without CMEs in accordance with the velocity and length of filaments. These lines can be expressed as

$$
\boldsymbol{w}^{\mathrm{T}} \boldsymbol{x}=w_{0}+w_{1} x_{1}+w_{2} x_{2}=0,
$$

where $\boldsymbol{w}=\left(w_{0}, w_{1}, w_{2}\right)^{\mathrm{T}}$ is a coefficient vector to be optimised, and $\boldsymbol{x}=\left(1, x_{1}, x_{2}\right)^{\mathrm{T}}$ is a feature vector, which corresponds to the observation. In our case, $x_{1}$ is a common

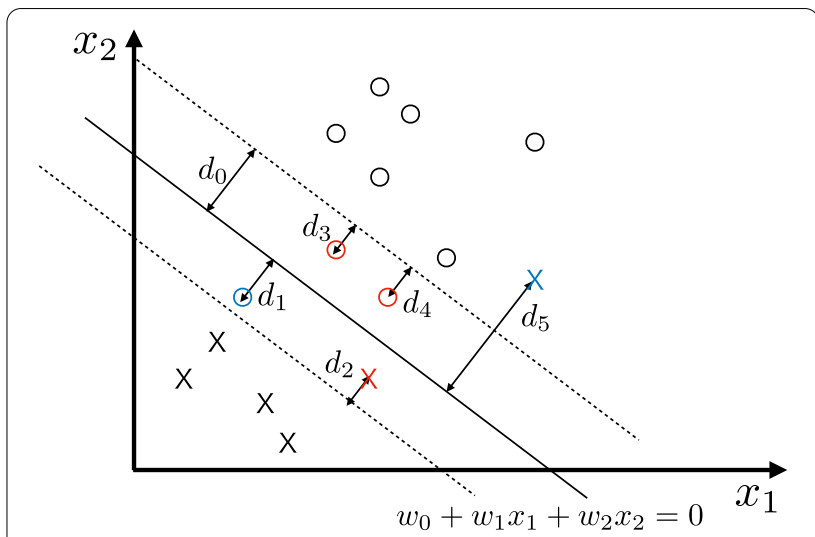

Fig. 6 The schematic view of LSVC in our case

$\log$ of a normalised velocity $\left(V_{r_{-} \max }, V_{\max }\right.$, or $V_{\text {pos }}$ divided by $V_{0}$ ), and $x_{2}$ is a common log of a normalised length ( $L$ divided by $L_{0}$ ).

We optimised $\boldsymbol{w}$ by minimising the loss, $l$, defined as

$$
l=\frac{1}{2} \boldsymbol{w}^{\mathrm{T}} \boldsymbol{w}+C \sum_{i=1}^{N}\left(\max \left(1-y^{i} \boldsymbol{w}^{\mathrm{T}} \boldsymbol{x}^{i}, 0\right)\right)^{2},
$$

where $i$ and $N$ are the index and the number of our selected events, $y^{i} \in\{-1,1\}$ is the label of the CME association for the event $i$ ( -1 : without CME, 1 : with CME), $\boldsymbol{x}^{i}=\left(1, x_{1}^{i}, x_{2}^{i}\right)^{\mathrm{T}}$ is an actual observed values for the event $i$, and $C$ is a constant (in our case, set to be 100). The first term in Eq. (9) is a penalty term, which prevents the classifier from overfitting to the sample. As for the second term, intuitively, to minimise it corresponds to (1) maximising the sum of the distances to the line from the correctly classified "near" data points and, simultaneously, (2) minimising the sum of the distances to the line from the misclassified data points.

Here, we describe the meaning of the second term in more detail. Figure 6 displays the schematic view of our analysis.

Suppose that we aim to divide open circles and crosses according to two variables, $x_{1}$ and $x_{2}$. On the basis of the present $\boldsymbol{w}$, we can calculate $d_{0}$ defined as

$$
d_{0}=\frac{1}{\sqrt{w_{1}^{2}+w_{2}^{2}}},
$$

and select the correctly classified "near" data points whose distances to the line are smaller than $d_{0}$, i.e., the correctly classified data points between the dashed lines in the figure (coloured in red). We also select the misclassified data points regardless of their distances to the line (coloured in blue). Then, Eq. (9) should be written as 


$$
l=\frac{1}{2} \boldsymbol{w}^{\mathrm{T}} \boldsymbol{w}+\frac{C}{d_{0}}\left\{\left(d_{1}+d_{5}+2 d_{0}\right)+\left(d_{2}+d_{3}+d_{4}\right)\right\} .
$$

The first parenthesis in Eq. (11) corresponds to the sum of the distances to the solid line from the misclassified events (and $d_{0}$ multiplied by the number of them). The second parenthesis sums up the distances to the nearest dashed line from the correctly classified "near" data points. Finally, by solving the minimisation of $l$ for $w$, we obtained the well separable lines shown in Fig. 1.

Received: 2 September 2020 Accepted: 10 February 2021

Published online: 26 February 2021

\section{References}

Al-Omari M, Qahwaji R, Colak T, Ipson S (2010) Machine leaning-based investigation of the associations between cmes and filaments. Sol Phys 262(2):511-539. https://doi.org/10.1007/s11207-010-9516-5

Ashman KM, Bird CM, Zepf SE (1994) Detecting bimodality in astrometrical datasets. Astron J 108:2348. https://doi.org/10.1086/117248

Boteler D (2001) Assessment of geomagnetic hazard to power systems in Canada. Nat Hazards 23(2-3):101-120

Brueckner G, Howard R, Koomen M, Korendyke C, Michels D, Moses J, Socker D, Dere K, Lamy P, Llebaria A et al (1995) The large angle spectroscopic coronagraph (LASCO). The SOHO mission. Springer, Dordrecht, pp 357-402

Carlson NL, Sultanova MR, Vithanage SPK, Barkhouse WA, Gamage GLIK, Rude CM, López-Cruz O (2018) Globular cluster population of the HST frontier fields galaxy j07173724+3744224. Mon Not R Astron Soc 480(2):19561972. https://doi.org/10.1093/mnras/sty1979

Fan RE, Chang KW, Hsieh CJ, Wang XR, Lin CJ (2008) Liblinear: a library for large linear classification. J Mach Learn Res 9:1871-1874

Gilbert HR, Holzer TE, Burkepile JT, Hundhausen AJ (2000) Active and eruptive prominences and their relationship to coronal mass ejections. Astrophys J 537(1):503-515. https://doi.org/10.1086/309030

Gilbert HR, Falco LE, Holzer TE, MacQueen RM (2006) Application of a new technique for deriving prominence mass from SOHO EIT Fe XII (19.5 $\mathrm{nm}$ ) absorption features. Astrophys J 641(1):606-610. https://doi. org/10.1086/500354

Gopalswamy N, Shimojo M, Lu W, Yashiro S, Shibasaki K, Howard R (2003) Prominence eruptions and coronal mass ejection: a statistical study using microwave observations. Astrophys J 586(1):562

Heinzel P, Schmieder B, Fárník F, Schwartz P, Labrosse N, Kotrč P, Anzer U, Molodij G, Berlicki A, DeLuca EE et al (2008) Hinode, TRACE, SOHO, and ground-based observations of a quiescent prominence. Astrophys J 686(2):1383-1396. https://doi.org/10.1086/591018

Hori K, Culhane J (2002) Trajectories of microwave prominence eruptions. Astron Astrophys 382(2):666-677

Ichimoto K, Ishii TT, Otsuji K, Kimura G, Nakatani Y, Kaneda N, Nagata S, UeNo S, Hirose K, Cabezas D et al (2017) A new solar imaging system for observing high-speed eruptions: solar dynamics doppler imager (SDDI). Sol Phys 292(4):63

Illing RME, Hundhausen AJ (1985) Observation of a coronal transient from 1.2 to 6 solar radii. J Geophys Res Space Phys 90(A1):275-282. https://doi. org/10.1029/JA090iA01p00275
Jing J, Yurchyshyn VB, Yang G, Xu Y, Wang H (2004) On the relation between filament eruptions, flares, and coronal mass ejections. Astrophys J 614(2):1054-1062. https://doi.org/10.1086/423781

Lemen JR, Akin DJ, Boerner PF, Chou C, Drake JF, Duncan DW, Edwards CG, Friedlaender FM, Heyman GF, Hurlburt NE et al (2011) The atmospheric imaging assembly (AIA) on the solar dynamics observatory (SDO). The solar dynamics observatory. Springer, New York, pp 17-40

McAllister AH, Dryer M, Mclntosh P, Singer H, Weiss L (1994) A large polar crown coronal mass ejection and a "problem" geomagnetic storm: April 14-23. J Geophys Res 101(A6):13497-13516. https://doi. org/10.1029/96JA00510

McAllister AH, Kurokawa H, Shibata K, Nitta N (1996) A filament eruption and accompanying coronal field changes on November 5, 1992. Sol Phys 169:123-149

McCauley PI, Su Y, Schanche N, Evans KE, Su C, McKillop S, Reeves KK (2015) Prominence and filament eruptions observed by the solar dynamics observatory: statistical properties, kinematics, and online catalog. Sol Phys 290(6):1703-1740

Morimoto T, Kurokawa H (2003) Eruptive and quasi-eruptive disappearing solar filaments and their relationship with coronal activities. Publ Astron Soc Jpan 55:1141-1151. https://doi.org/10.1093/pasj/55.6.1141

Muratov AL, Gnedin OY (2010) Modelling the metallicity distribution of globular clusters. Astrophys J 718(2):1266-1288. https://doi. org/10.1088/0004-637x/718/2/1266

Nakajima H, Nishio M, Enome S, Shibasaki K, Takano T, Hanaoka Y, Torii C, Sekiguchi H, Bushimata T, Kawashima S, Shinohara N, Irimajiri Y, Koshiishi H, Kosugi T, Shiomi Y, Sawa M, Kai K (1994) The Nobeyama radioheliograph. IEEE Proc 82(5):705-713

Parenti S (2014) Solar prominences: observations. Living Rev Sol Phys. https:// doi.org/10.12942//rsp-2014-1

Pojoga S, Huang T (2003) On the sudden disappearances of solar filaments and their relationship with coronal mass ejections. Adv Space Res 32(12):2641-2646. https://doi.org/10.1016/s0273-1177(03)00936-0

Seki D, Otsuji K, Isobe H, Ishii TT, Sakaue T, Hirose K (2017) Increase in the amplitude of line-of-sight velocities of the small-scale motions in a solar filament before eruption. Astrophys J Lett 843(2):L24

Seki D, Otsuji K, Ishii TT, Hirose K, Iju T, UeNo S, Cabezas DP, Asai A, Isobe H, Ichimoto K, Shibata K (2019a) Smart/SDDI filament disappearance catalogue. Sun Geosph 14(2):95-103

Seki D, Otsuji K, Isobe H, Ishii TT, Ichimoto K, Shibata K (2019b) Small-scale motions in solar filaments as the precursors of eruptions. Publ Astron Soc Jpn 71(3):56

UeNo S, Nagata S, Kitai R, Kurokawa H (2004) Features of solar telescopes at the Hida observatory and the possibilities of coordinated observations with SolarB. In: Sakurai T, Sekii T (eds) The solar-B mission and the forefront of solar physics, vol 325. Astronomical Society of the Pacific Conference Series. Astronomical Society of the Pacific, San Francisco, p 319

Webb DF (2015) Eruptive prominences and their association with coronal mass ejections. Solar prominences. Springer, Cham, pp 411-432

Yashiro S, Gopalswamy N, Michalek G, St. Cyr OC, Plunkett SP, Rich NB, Howard RA (2004) A catalog of white light coronal mass ejections observed by the SOHOspacecraft. J Geophys Res Space Phys 109(A7):A07105. https:// doi.org/10.1029/2003JA010282

\section{Publisher's Note}

Springer Nature remains neutral with regard to jurisdictional claims in published maps and institutional affiliations. 